\title{
Dyslipidemia promotes germinal center reactions via IL-27
}

\author{
Heeju Ryu ${ }^{1,2}$ \& Yeonseok Chung ${ }^{1,2, *}$ \\ ${ }^{1}$ Laboratory of Immune Regulation, Institute of Pharmaceutical Sciences, Seoul National University, Seoul 08826, ${ }^{2}$ BK21 Plus Program, \\ College of Pharmacy, Seoul National University, Seoul 08826, Korea
}

\begin{abstract}
Cardiovascular disease such as atherosclerosis is caused by imbalanced lipid metabolism and represents a leading cause of death worldwide. Epidemiological studies show that patients with systemic autoimmune diseases exhibit a higher incidence of atherosclerosis. Conversely, hyperlipidemia has been known to accelerate the incidence of autoimmune diseases in humans and in animal models. However, there is a considerable gap in our understanding of how atherosclerosis impacts the development of the autoimmunity in humans, and vice versa. The atherosclerosis-related autoimmune diseases include psoriasis, rheumatoid arthritis, systemic lupus erythematosus (SLE) and diabetes mellitus. By using animal models of atherosclerosis and SLE, we have recently demonstrated that hyperlipidemia significantly accelerates the development of autoantibodies, by inducing autoimmune follicular helper $T\left(T_{F H}\right)$ cells. Mechanistic studies have identified that hyperlipidemia induces IL-27 production in a TLR4-dependent manner, likely via downregulating LXR expression in dendritic cells. In this case, mice lacking IL-27 do not develop enhanced antibody responses. Thus it is noted that these findings propose a mechanistic insight responsible for the tight association between cardiovascular diseases and SLE in humans. [BMB Reports: Perspective 2018; 51(8): 371-372]
\end{abstract}

Atherosclerosis-related coronary and cerebral artery diseases represent the leading cause of death in the United States, and are known to cause more than $25 \%$ of the deaths. The

*Corresponding author. E-mail: yeonseok@snu.ac.kr

https://doi.org/10.5483/BMBRep.2018.51.8.171

\section{Received 7 July 2018}

Keywords: Autoantibodies, Autoimmune lupus, Germinal center reaction, IL-27, $\mathrm{T}_{\mathrm{FH}}$ cell

Abbreviations: ApoE, Apolipoprotein E; DC, Dendritic cell; GC, Germinal center; IFN- $\gamma$, Interferon- $\gamma$; LXR, Liver X receptor; SLE, Systemic lupus erythematosus; $\mathrm{T}_{\mathrm{FH}}$ cell, Follicular helper $\mathrm{T}$ cell; TLR4, Toll-like receptor 4; WT, wild-type

Perspective to: Heeju Ryu et al. Nature Immunology 19, 583-593 (2018), doi: 10.1038/s41590-018-0102-6 underlying pathogenesis for this condition includes a characteristically imbalanced lipid metabolism that leads to hyperlipidemia, as well as inappropriate immune responses that affect the arterial vasculature. In addition, preclinical and clinical observations clearly demonstrate an essential pathogenic involvement of innate and adaptive immunity in atherosclerosis. For instance, blockade of leukocyte migration by targeting chemokine receptors (eg. CCR2, CCR5) significantly ameliorates atherosclerosis in studied animal models. Moreover, mice lacking the $\mathrm{T}_{\mathrm{H}} 1$ transcription factor T-bet, IFN- $\gamma$ or the IFN- $\gamma$ receptor are resistant to atherosclerosis. Therefore, an aberrant activation of inflammatory innate and adaptive immune responses seems to contribute to the pathogenesis of atherosclerosis (Hansson et al (2011) Nat Immunol 12, 204-212). Conversely, an effect of atherogenic factors on the innate immune system has been reported. For instance, modified LDL particles stimulate macrophages to produce IL-1 through MyD88 or inflammasome (Duewell et al (2010) Nature 464, 1357-1361), leading to a pro-inflammatory microenvironment. On the other hand, few studies to date have addressed the possible contribution of atherogenic factors to shape adaptive the $\mathrm{T}$ cell responses in humans. Importantly, numerous epidemiologic studies indicate a strong incidental correlation between atherosclerosis and chronic autoimmune disorders, such as rheumatoid arthritis (RA), psoriasis, systemic lupus erythematosus (SLE), all of which are mediated by aberrantly activated self-reactive $\mathrm{T}$ cells. Moreover, treatment of hyperlipidemia such as statins and low-fat diet leads to clinical improvement in patients diagnosed with psoriasis, indicating a potential role of hyperlipidemia in the pathogenesis of autoimmune diseases. Although current epidemiologic and clinical observations strongly suggest a higher risk of T cell-mediated autoimmune diseases in patients with atherosclerosis, little is known about the underlying mechanism by which these atherogenic factors modulate autoimmune $\mathrm{T}$ cell responses. In this regard, our previous study identified that autoreactive $T_{H} 17$ cells as a cellular linker between atherosclerosis and animal models of experimental autoimmune encephalomyelitis (Lim et al (2014) Immunity 40, 153-165). Among the atherosclerosis- related autoimmune diseases, psoriasis is mainly mediated by the noted $T_{H} 17$ cell responses. However, the $T_{H} 17$ cell is unlikely the major pathogenic T cell in SLE; rather it is noted that the follicular helper $\mathrm{T}\left(\mathrm{T}_{\mathrm{FH}}\right)$ cell has been proposed to be a

ISSN: 1976-670X (electronic edition)

Copyright (c) 2018 by the The Korean Society for Biochemistry and Molecular Biology

(c) This is an open-access article distributed under the terms of the Creative Commons Attribution Non-Commercial License (http://creativecommons.org/licenses/by-nc/4.0) which permits unrestricted non-commercial use, distribution, and reproduction in any medium, provided the original work is properly cited. 


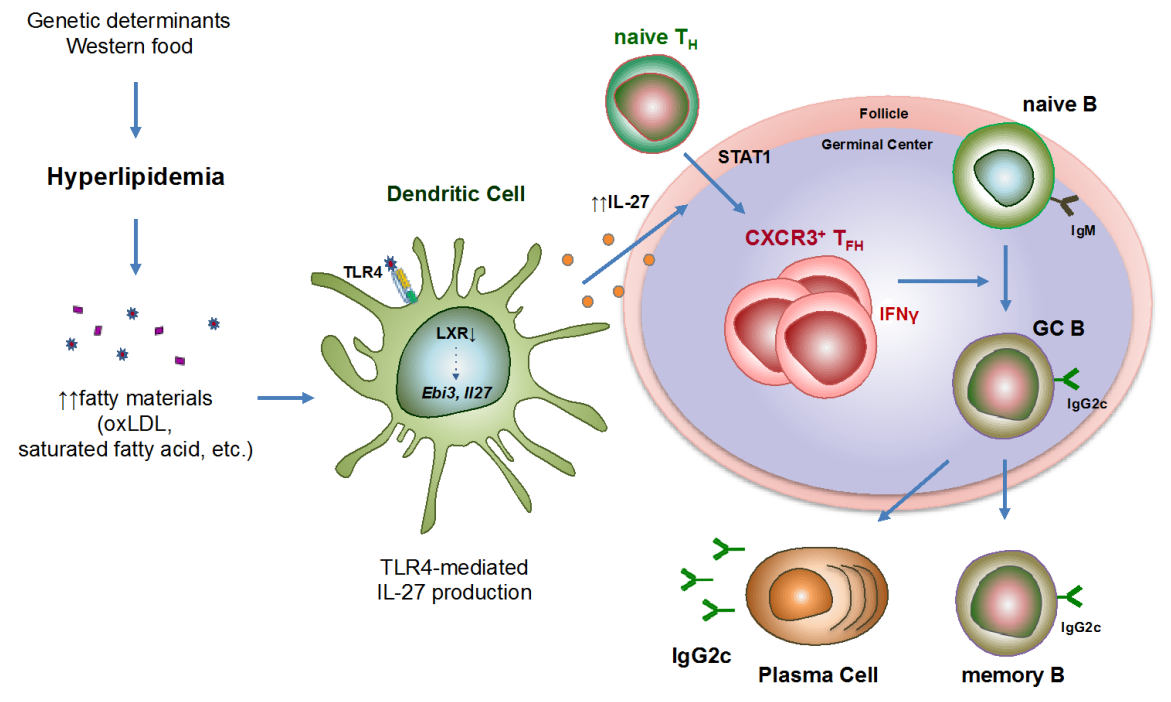

Diagram 1. Graphic summary of the study. Hyperlipidemic environment triggers IL-27 secretion by dendritic cells in a TLR4- and LXR-dependent manner, which then stimulates the differentiation of $\mathrm{CXCR}^{+} \mathrm{T}_{\mathrm{FH}}$ cells. These CXCR3 ${ }^{+} \mathrm{T}_{\mathrm{FH}}$ cells induce germinal center reactions and the production of pathogenic $\operatorname{lgG} 2 \mathrm{C}$ autoantibodies to aggravate autoimmune lupus in mice. pathogenic helper T cell in antibody-mediated autoimmune diseases such as SLE.

To demonstrate the effect of hyperlipidemia on the pathogenesis of SLE, bone marrow cells from lupus-prone BXD2 mice were transferred into atherogenic Apoe ${ }^{-1-}$ mice $\left(A p o E^{\mathrm{BXD} 2}\right)$ or into $\mathrm{WT}\left(\mathrm{WT}^{\mathrm{BXD} 2}\right)$ mice as a control, and those mice were fed on a high-fat diet. Equally important to note is that the atherogenic recipient mice exhibited more profound lupus-like phenotype, such as the glomerulonephritis and high levels of autoantibodies, particularly notable was a large increase in the pathogenic IgG2c isotype. Essentially, the $\mathrm{T}_{\mathrm{FH}}$ cells are one of the $\mathrm{CD}^{+}{ }^{+} \mathrm{T}$ cell subsets that provide help to the $\mathrm{B}$ cells for antibody production, and are essential for the germinal center (GC) reactions (Crotty et al (2014) Immunity 41, 529-542). The frequency and number of cells in $T_{F H}$ cells, GC B cells, and plasma cells population was significantly higher in $A p o E^{B X D 2}$ than in $\mathrm{WT}^{\mathrm{BXD2}}$. Among $\mathrm{T}_{\mathrm{FH}}$ cell subsets, $\mathrm{CXCR}^{+} \mathrm{T}_{\mathrm{FH}}$ cell population was significantly enriched in $A p o E^{\mathrm{XX} 2}$, while changes in other subpopulation were noted as considered marginal. When $\mathrm{T}_{\mathrm{FH}}$ cells from those mice were co-cultured with naive B cells, atherogenic $\mathrm{T}_{\mathrm{FH}}$ cells were far more potent in inducing IgG production, particularly IgG2c isotype, which were attenuated by neutralization of IFN- $\gamma$. RNA-seq data of $\mathrm{T}_{\mathrm{FH}}$ cells from Apoe $^{-/-}$and WT mice revealed that genes associated with inflammatory responses, SLE and IFN- $\gamma$ related pathway as well as genes associated with $B$ cell help capability were highly enriched in the cells from atherogenic mice. In search of a cellular and molecular mechanism, we found that serum levels of IL-27 were significantly increased in the atherogenic mice compared with the WT mice. In the same fashion, bone marrow reconstitution experiment demonstrated that hyperlipidemia induced IL-27 in a TLR4-dependent manner. Notably, the expression of the liver
$X$ receptor $(L X R) \beta$ was decreased in the DCs isolated from the atherogenic mice, and the restoration of the LXR activity reduced IL-27 production by atherogenic DCs, suggesting that the TLR4 and LXR regulate IL-27 secretion by DCs in atherogenic mice. In addition, the IL-27 is a heterodimeric cytokine that has been shown to mediate $\mathrm{T}_{\mathrm{FH}}$ cell differentiation (Batten et al (2010) J Exp Med 207, 2895-2906). In the absence of IL-27 signal, Apoe ${ }^{-/-}$mice did not exhibit augmented germinal center reactions, $\mathrm{CXCR}^{+} \mathrm{T}_{\mathrm{FH}}$ population, or $\operatorname{lgG} 2 \mathrm{c}$ antibody production, while the frequency of follicular regulatory $\mathrm{T}\left(\mathrm{T}_{\mathrm{FR}}\right)$ cells were elevated (Diagram 1). Importantly, we also found that patients with hypercholesterolemia exhibited increased serum levels of IL-27, autoantibodies, and IgG1 and IgG3 antibodies, which is characteristically a homolog of IgG2c in mice, compared with healthy controls, indicating that a 'hyperlipidemia-IL-27-IgG2 $\mathrm{c}^{\prime}$ axis found in mice might be applicable to humans as well.

Collectively, our study provides a novel mechanistic insight into the tight association of atherosclerosis and antibodymediated autoimmune diseases such as SLE. Hyperlipidemia promotes the secretion of IL-27 from CD $11 \mathrm{~b}^{+}$dendritic cells in a TLR4-dependent manner. This cytokine signals through STAT1 to generate $\mathrm{CXCR}^{+}{ }^{+} \mathrm{T}_{\mathrm{FH}}$ cells, and enhances subsequent autoimmune germinal center reactions to generate pathogenic IgG2c autoantibodies. Thus, IL-27 might be a potential therapeutic target to treat atherosclerosis-associated SLE in humans.

\section{ACKNOWLEDGEMENTS}

This work is supported by a grant numbered 2017R1A2B 3007392 from the National Research Foundation of Korea. 